\title{
APORTE FISCAL INDIRECTO: UNA PROPUESTA PARA SU MODERNIZACIÓN
}

\author{
Lorena Flores, Javier González, \\ Jorge Rodríguez y Daniela Sugg ${ }^{1}$
}

\begin{abstract}
RESUMEN
El Aporte Fiscal Indirecto (AFI) fue creado en 1981 para aumentar la calidad de la educación superior mediante la competencia entre las instituciones por captar a los mejores alumnos. Con el tiempo este potencial generador de incentivos ha decaído, pues hoy el AFI solo representa el 3,5\% del aporte fiscal total para educación superior. A esto se suman varias debilidades en su diseño. El presente trabajo plantea propuestas de perfeccionamiento del AFI para que constituya una herramienta efectiva para promover la equidad, calidad y eficiencia del sistema de educación superior. Entre ellas se destaca: aumentar su peso relativo como fuente de financiamiento; exigir que las instituciones beneficiarias estén acreditadas para poder recibirlo; considerar el ranking de egreso de enseñanza media para la identificación de los alumnos causantes del beneficio; reservar cupos AFI para carreras técnicas; corregir el valor del AFI por el nivel socioeconómico del alumno y pagarlo en cuotas para incentivar la retención y titulación oportuna de los alumnos.
\end{abstract}

Palabras clave: educación superior; financiamiento público; Aporte Fiscal Indirecto (AFI); Prueba de Selección Universitaria (PSU).

\section{INDIRECT FISCAL CONTRIBUTION: A PROPOSAL TO UPGRADE IT}

\section{ABSTRACT}

The Indirect Fiscal Contribution (AFI, for the Spanish acronym) was created in 1981 in order to improve the quality of higher education through the competence among institutions to recruit best students. Over time, this potential source of incentives has become weak, as today AFI only represents 3.5 per cent of the total fiscal contribution for higher education. Moreover, it shows several weaknesses in design. This work provides proposals to mainstream the AFI to make it an effective tool to promote equity, quality and efficiency of the higher education system. Among them, it highlights: to improve its relative weight as a funding source; to demand the beneficiary institutions to be accredited to receive it; to consider the secondary school graduation ranking to identify students generating the benefit; to reserve AFI places for technical careers; to correct the AFI value by the students SES and to pay it monthly to improve the retention and timely graduation of students.

Keywords: higher education; public funding; Indirect Fiscal Contribution (AFI); university entry test (PSU).

1 Investigadores independientes. Santiago. Chile. Contacto: lore.flores@gmail.com 


\section{APORTE FISCAL INDIRECTO: UNA PROPUESTA PARA SU MODERNIZACIÓN}

\section{Introducción ${ }^{2}$}

Contar con profesionales y técnicos de calidad es clave para el crecimiento económico y social de un país, por lo que invertir en educación es indispensable para alcanzar el desarrollo. En el ámbito de la educación superior -profesional y técnica- existe un rol para el Estado, referido principalmente a incentivar que se alcance el nivel óptimo social de inversión, remover restricciones a su financiamiento y acceso, generar información para mejorar las decisiones involucradas e incentivar la producción de bienes públicos en investigación y docencia.

En los últimos años el Estado chileno ha aumentado significativamente su inversión en educación superior, mediante instrumentos de financiamiento tanto a las instituciones como a los estudiantes. Es así como el aporte fiscal para educación superior pasó de 268.135 millones de pesos en el año 2000 a un presupuesto de 635.990 millones de pesos en 2010, lo que implica que en diez años se duplicó en términos reales.

Ello ha incidido en que entre 2000 y 2009 la matrícula total en educación superior de pregrado haya crecido un $91,6 \%$, destacándose en particular el aumento de la matrícula de alumnos de los tres quintiles de menores ingresos (Rodríguez et al, 2010). Según la Organización para la Cooperación y Desarrollo Económicos (OCDE), esta evolución ha implicado pasar de una educación terciaria de elite a una educación terciaria masiva (OCDE y Banco Mundial, 2009).

Esta nueva realidad implica una serie de desafíos para el sistema de educación superior chileno, como lo son la calidad de la educación

2 Los autores agradecen los valiosos comentarios de José Joaquín Brunner, los miembros del Programa Anillo de Investigación en Políticas de Educación Superior de la Universidad Diego Portales, Tania Hernández y María Olivia Recart. 
impartida, la equidad de acceso y retención, y la eficiencia, focalización y sustentabilidad de la inversión pública en el sector.

En este contexto, el presente trabajo tiene como finalidad analizar perfeccionamientos al Aporte Fiscal Indirecto (AFI) para que constituya una herramienta efectiva para contribuir a aumentar la equidad, calidad y eficiencia del sistema de educación superior chileno. Ciertamente, alcanzar estas cualidades en el sistema requiere revisar también los restantes instrumentos de financiamiento de la educación superior, los que en su mayoría fueron diseñados para una realidad diferente, en la que la cobertura era mucho menor que la actual. En consecuencia, análisis similares al del presente trabajo son necesarios también para los restantes instrumentos de aporte fiscal a la educación superior, tanto los dirigidos a los estudiantes como los dirigidos a las instituciones, con el objetivo de avanzar en la construcción de un sistema integrado y coherente de financiamiento del sistema. Con ello en mente, en el presente trabajo sugerimos perfeccionar el AFI debido a que su diseño actual tiene elementos con potencial para generar incentivos virtuosos en las instituciones de educación superior, que permiten orientar al sistema en la dirección deseada.

Este instrumento fue creado en 1981, con el objetivo de aumentar la calidad de la docencia de pregrado mediante la promoción de la competencia entre las instituciones de educación superior por captar a los mejores alumnos. Sin embargo, el AFI hoy no genera un incentivo lo suficientemente poderoso debido al poco peso relativo que representa, el que alcanza alrededor de $11 \%$ del aporte fiscal para las instituciones y solo un 3,5\% del aporte fiscal total para educación superior. Esta pérdida de influencia se refleja también en que en la década de los 80 el AFI cubría en promedio a cerca del 40\% de la matrícula de alumnos de primer año, mientras que en 2009 este porcentaje cayó a alrededor del 10\%.

Por tanto, para ser efectivo, el AFI debe aumentar su peso relativo como fuente de financiamiento de la educación superior, pagando más por estudiante y aumentando su cobertura de alumnos. Pero ello necesariamente debe ir de la mano de un rediseño que refuerce su 
potencial generador de incentivos virtuosos, mediante el uso de un adecuado predictor de rendimiento académico futuro de los estudiantes, el fomento de la retención de los alumnos, el establecimiento de requisitos de calidad para las instituciones que lo captan, el evitar sesgos en contra de los alumnos de menor nivel socioeconómico, el relacionar sus aportes con los costos de educar, y el adaptarse a la trayectoria de los estudiantes de educación media técnico profesional.

Para el análisis del AFI, en la sección 2 del documento se describe su funcionamiento y su inserción en el esquema de financiamiento del sistema de educación superior chileno. En la sección 3 se realiza una evaluación de su diseño actual. En la sección 4 se presenta una propuesta para su perfeccionamiento. Finalmente, en la sección 5 se concluye sobre los lineamientos de política a seguir, mientras que como anexo se presenta, a modo ilustrativo, una simulación particular de propuesta de perfeccionamiento del AFI.

\section{Descripción del Aporte Fiscal Indirecto}

\subsection{Funcionamiento}

El AFI se estableció en el DFL No 4 de 1981 y su aplicación comenzó en $1982^{3}$. Fue concebido con el objetivo de mejorar la calidad de la docencia de pregrado, mediante la promoción de la competencia entre las instituciones de educación superior por captar a los mejores alumnos que ingresan al sistema terciario (Brunner, 2005). Al AFI pueden acceder las universidades, institutos profesionales y centros de formación técnica que sean reconocidos por el Estado como instituciones de educación superior.

El monto total del AFI es determinado por la Ley de Presupuestos del Sector Público de cada año ${ }^{4}$. Su distribución entre las instituciones

3 Luego fue modificado en el artículo 20 de la Ley $N^{\circ} 18.681$ de 1987, donde quedó establecido su actual mecanismo de distribución, y en el artículo 51 de la Ley $N^{\circ} 18.768$ de 1988, el que extendió el beneficio a los institutos profesionales y centros de formación técnica, además de establecer como límite de cobertura a los 27.500 mejores puntajes de la Prueba de Aptitud Académica (PAA), posteriormente sustituida por la Prueba de Selección Universitaria (PSU). Los aspectos de procedimientos operativos del AFI están contenidos en el Reglamento del 4 de mayo de 1982.

4 Para 2010 la Ley de Presupuestos contempló 188 millones de pesos para el AFI. 
se realiza en función de donde se matriculen los 27.500 mejores puntajes de la Prueba de Selección Universitaria (PSU) de cada año ${ }^{5}$, generando un mayor monto de AFI los alumnos de mejores puntajes. En particular, los 27.500 matriculados se ordenan de menor a mayor puntaje en cinco grupos de aproximadamente 5.500 alumnos cada uno. Los grupos se ajustan por un factor de ponderación que toma valores de 1, 3, 6, 9 y 12, donde el ponderador mayor es para el grupo con más altos puntajes PSU. Así, un alumno del grupo de mayor puntaje PSU genera 12 veces más AFI para la institución de educación superior donde se matricula, que un alumno del primer grupo.

La tabla 1 muestra los cinco tramos AFI vigentes para 2010, con sus respectivos puntajes PSU y el monto de recursos que genera cada alumno causante. Para 2010 el valor de la unidad AFI fue de 123.536 pesos, por lo que un alumno del grupo de mayor puntaje aportó a la institución de educación superior en que se matriculó un total de 1.483.438 pesos, mientras que un alumno del grupo de menor puntaje aportó 123.536 pesos.

Tabla 1. Tramos AFI, proceso 2010

\begin{tabular}{|c|c|c|c|c|c|}
\hline \multirow{2}{*}{ Tramo AFI } & \multicolumn{2}{|c|}{ Puntaje PSU } & \multirow{2}{*}{$N^{\circ}$ alumnos por tramo } & \multirow{2}{*}{ Ponderador } & \multirow{2}{*}{ Monto por alumno } \\
\cline { 2 - 3 } & Desde & Hasta & & & \\
\hline 1 & 616,5 & 631,0 & 5.451 & 1 & $\$ 123.536$ \\
\hline 2 & 631,5 & 648,0 & 5.384 & 3 & $\$ 370.609$ \\
\hline 3 & 648,5 & 670,0 & 5.620 & 6 & $\$ 741.219$ \\
\hline 4 & 670,5 & 703,0 & 5.542 & 9 & $\$ 1.111 .828$ \\
\hline 5 & 845,5 & 703,5 & 5.526 & 12 & $\$ 1.483 .438$ \\
\hline
\end{tabular}

Fuente: División de Educación Superior, Ministerio de Educación.

Cabe mencionar que la norma original del DFL No 4 de 1981, derogada en 1987, establecía un factor de ponderación adicional de 2,5 en el caso de alumnos que se matriculasen en Medicina y Odontología; de 1,8 para aquellos alumnos que se matriculasen en Bioquímica, Ingeniería Civil, Agronomía, Ingeniería Forestal, Química y Farmacia y Medicina Veterinaria; y de 1 para los que se matriculasen en otras carreras o programas de estudios superiores. Por tanto, se puede suponer que originalmente un objetivo adicional del AFI era

5 Considerando el promedio de las pruebas de matemáticas y de lenguaje y comunicación. Originalmente se consideraban los puntajes de la PAA. 
incentivar la formación profesional en áreas consideradas como prioritarias, y/o subsidiar carreras más costosas.

\subsection{El AFI en el contexto del sistema de educación superior chileno}

El AFI es uno de los varios instrumentos con que el Estado de Chile contribuye actualmente al financiamiento de la educación superior. El aporte fiscal total para educación superior contó con un presupuesto de 635.990 millones de pesos para 2010, lo que implica que en los últimos diez años aumentó un 137\% en términos reales. Así, en 2010 el aporte fiscal para educación superior representó un 14,2\% del gasto público en educación (1,3 puntos porcentuales más de lo que representó en el año 2000) y fue equivalente a 2,7\% del gasto público total $(0,5 \text { puntos porcentuales más que en el año 2000 })^{6}$.

Este aporte fiscal para educación superior se divide entre los recursos que se asignan a las instituciones de educación superior (financiamiento de la oferta) y los que se asignan a los estudiantes (financiamiento de la demanda). En el año 2000 estos últimos representaron el 28,7\% del aporte fiscal para educación superior (porcentaje levemente superior al 25,7\% que representaron en 1990), participación que aumentó significativamente a $68,0 \%$ en 2010 (Gráfico 1), siendo sus componentes más relevantes los créditos y las becas de arancel.

6 Cifras estimadas sobre la base de información de la División de Educación Superior del Ministerio de Educación, de la Dirección de Presupuestos del Ministerio de Hacienda y de la Ley de Presupuestos del Sector Público para el año 2010. 
Gráfico 1. Composición del aporte fiscal para educación superior 1990, 2000 y 2010

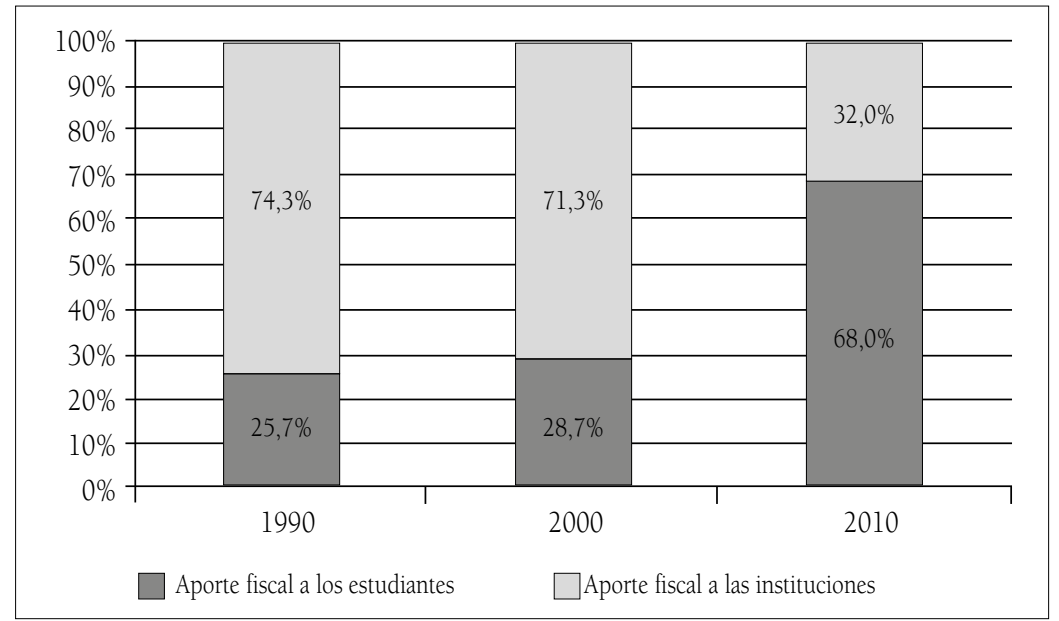

Fuente: División de Educación Superior del Ministerio de Educación para años 1990 y 2000 y Ley de Presupuestos del Sector Público para el año 2010.

En cuanto al aporte fiscal a las instituciones de educación superior, este creció un 6\% real en el período 2000-2010, pasando de 191.300 millones de pesos en el año 2000 a 203.524 millones de pesos en 2010 (en pesos de 2010). El componente más importante es el Aporte Fiscal Directo (AFD), que representa un 68,7\% promedio en el período. La comparación con 1990 muestra una leve disminución del peso relativo del AFD y una disminución importante de la participación relativa del AFI, a costa del surgimiento de los Fondos para Fortalecimiento Institucional y Otros (programas especiales) (gráfico 2).

Así, se verifica que el AFI representó en 2010 alrededor de $11 \%$ del aporte fiscal para las instituciones de educación superior, constituyendo de esta manera cerca de un 3,5\% del aporte fiscal total para educación superior. En contraste, en los años 90, a casi diez años de su creación, representaba más del 24\% del aporte fiscal para las instituciones de educación superior y aproximadamente un 18,2\% del aporte fiscal total para educación superior. 
Gráfico 2. Composición del aporte fiscal a las instituciones de educación superior 1990, 2000 y 2010

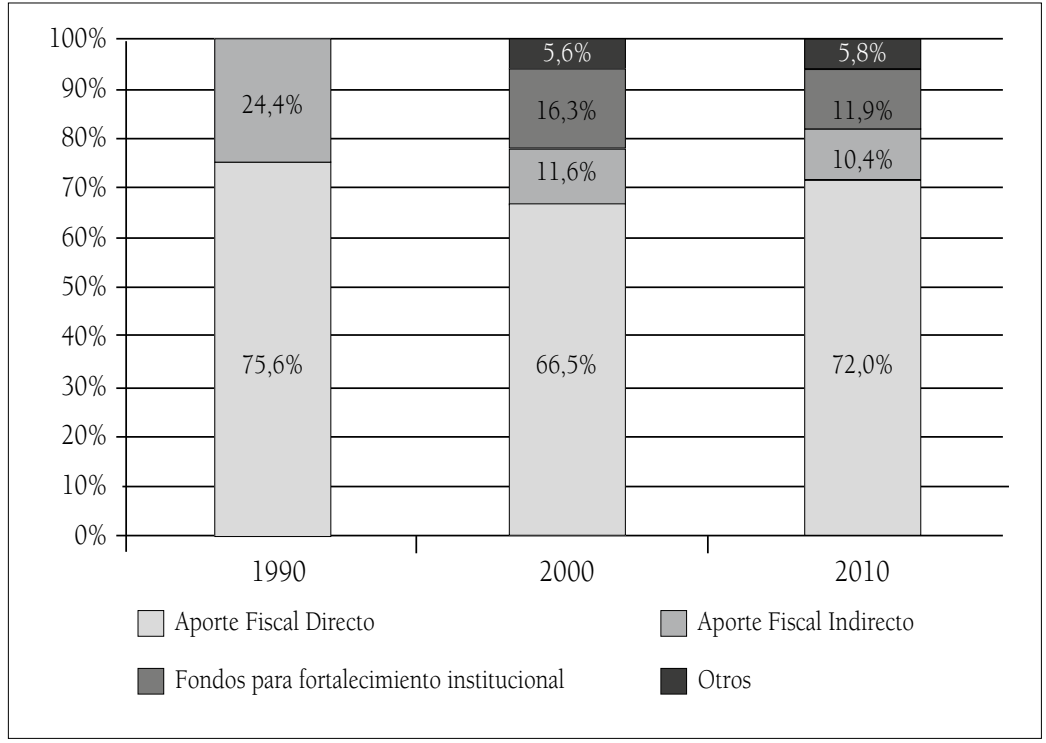

Fuente: División de Educación Superior del Ministerio de Educación para años 1990 y 2000 y Ley de Presupuestos del Sector Público para el año 2010.

La pérdida de peso relativo del AFI se debe a que se han priorizado otras fuentes de aporte fiscal para la educación superior. Pero en comparación con 1990, también se advierte una disminución en términos absolutos de los recursos canalizados mediante el AFI. Es así como para 2010 se presupuestaron 21.188 millones de pesos ${ }^{7}$, mientras que para 1990 se presupuestó la asignación de 24.817 millones de pesos (ambas cifras en pesos de 2010) (Gráfico 3).

7 Cabe señalar que para 2010, como una medida de cierta forma complementaria al AFI, se presupuestaron 142 millones de pesos para las instituciones de educación superior que matriculasen a alumnos egresados el año anterior, pertenecientes a los cuatro primeros quintiles de menores ingresos, y con un ranking de notas de enseñanza media dentro del $5 \%$ mejor de su respectivo establecimiento educacional. 
Gráfico 3. Evolución de recursos del AFI (en millones de pesos de 2010)

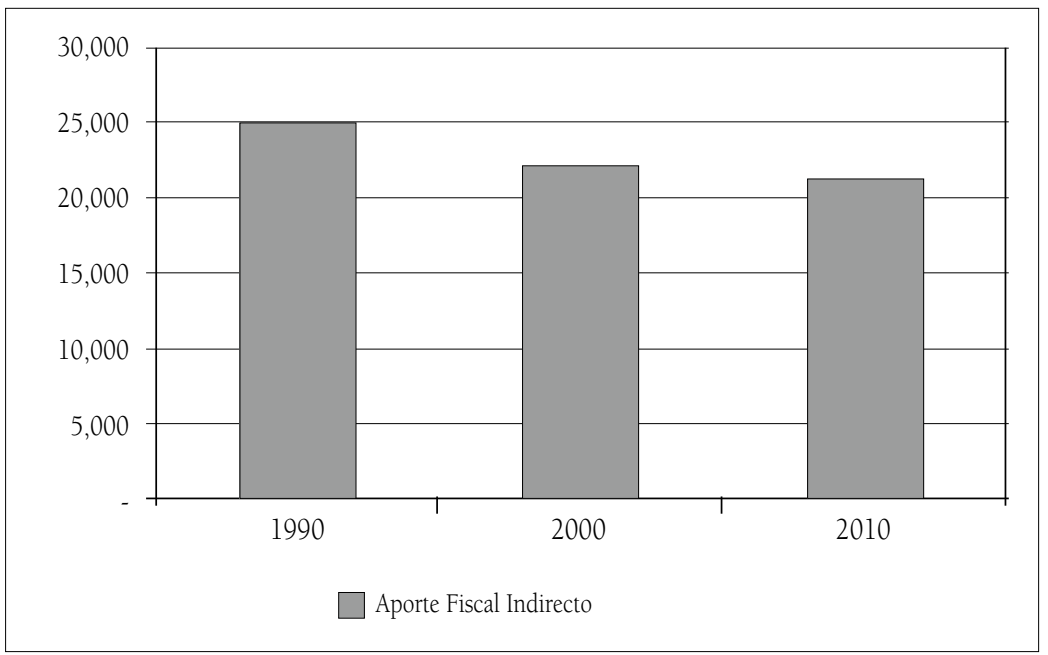

Fuente: División de Educación Superior del Ministerio de Educación para años 1990 y 2000 y Ley de Presupuestos del Sector Público para el año 2010.

En términos de cobertura de alumnos, como se ha señalado, la distribución del AFI se realiza en función de dónde se matriculan los 27.500 mejores puntajes de la PSU. Ello representó aproximadamente el $10 \%$ de la matrícula de alumnos de primer año en 2009. Esto contrasta con la situación de principios de la década de los 80, cuando se creó el AFI, época en que los 27.500 mejores puntajes representaban cerca del $40 \%$ de la matrícula de alumnos de primer año. Esto también es un indicio de la pérdida de importancia relativa que ha tenido el AFI como mecanismo de aporte fiscal a la educación superior.

En términos de las instituciones que acceden al AFI, pueden optar a él todas las universidades, institutos profesionales y centros de formación técnica reconocidos oficialmente por el Estado como instituciones de educación superior. En 2010 estos totalizaban 180, aunque en el proceso 2009 solo obtuvieron recursos del AFI 81 de ellos $^{8}$. En contraste, cuando se creó el AFI a principios de los años 80, podían optar a él un total de 25 instituciones. Esto podría ser señal de la mayor competencia potencial que existiría hoy por captar el AFI.

8 De acuerdo con información de la División de Educación Superior del Ministerio de Educación. 
Sin embargo, en 2009 el 57\% de los recursos por este concepto fue asignado a un conjunto de solo cinco instituciones (la Universidad de Chile, la Pontificia Universidad Católica de Chile, la Universidad de Santiago de Chile, la Universidad de Concepción y la Universidad Técnica Federico Santa María). Más en general, se verifica que los recursos del AFI son mayoritariamente captados por las universidades del Consejo de Rectores (77,1\% en 2009), seguidas por el resto de las universidades ( $21,5 \%$ en 2009), aunque este último porcentaje ha tendido a aumentar en años recientes (creció 5,4 puntos porcentuales entre 2005 y 2009).

\section{Aspectos clave del AFI}

El AFI posee fortalezas y debilidades como instrumento de financiamiento público de la educación superior. En esta sección se analizan ambas, considerando el contexto en el que opera, dado por un sistema de educación terciario que se rige principalmente por mecanismos de mercado, y en el que los recursos que aportan las familias tienen un peso relativo muy significativo en el financiamiento total del sistema ${ }^{9}$, lo que acota el efecto de los incentivos que generan las intervenciones públicas.

\subsection{Principales fortalezas del AFI}

Por su diseño, el AFI tiene potencial para generar incentivos virtuosos en las instituciones de educación superior mediante la generación de una mayor competencia. Según Rojas y Bernasconi (2002) existe una alta correlación entre la captación del AFI y la calidad de las instituciones, medido mediante el ranking nacional elaborado por revista Qué Pasa. En este sentido, que el mecanismo de asignación sea $100 \%$ competitivo representa una fortaleza respecto de otros mecanismos, como el AFD, que entregan recursos por razones históricas en vez de premiar conductas deseadas ${ }^{10}$.

9 De acuerdo con la OCDE (2009), en Chile las familias aportan el 82,5\% de los recursos del sistema de educación superior, lo que contrasta con el promedio de la OCDE en donde dicho aporte es del $27,4 \%$.

10 Un 95\% del AFD se distribuye según razones históricas y solo el 5\% del mismo se distribuye según indicadores relacionados con el desempeño. 
Otra fortaleza del AFI es que está abierto a todas las instituciones de educación superior reconocidas por el Estado (universidades sin distinguir dependencia, institutos profesionales y centros de formación técnica) y no solo a las instituciones pertenecientes al Consejo de Rectores de las Universidades Chilenas (CRUCH), como sucede con el AFD o la mayor parte de los recursos otorgados mediante MECESUP (Programa de Mejoramiento de la Calidad y Equidad de la Educación Superior).

Un tercer aspecto positivo del AFI se refiere a su contribución a uniformar y simplificar el mecanismo de admisión a las universidades. Esta tendencia se explica porque en la práctica se le ha impuesto un costo, en términos de una menor probabilidad de acceder al AFI, a todas aquellas instituciones que utilicen métodos alternativos de selección. Este fenómeno se hace evidente al observar que si bien en teoría solo las universidades del CRUCH deberían utilizar la PSU (pues esta es controlada y elaborada en el marco de dicho Consejo), en la práctica también la usan las universidades privadas, las cuales representan más del $50 \%$ de la matrícula universitaria ${ }^{11}$ y no poseen ninguna obligación que las comprometa. Cabe señalar que este aspecto positivo del AFI no quita que el instrumento específico cuyo uso ha contribuido a difundir (en un principio la PAA y luego PSU) tenga defectos que requieran ser mejorados.

Debido a estas fortalezas, el AFI es un instrumento con potencial. Sin embargo, en función de las debilidades que se describen a continuación, requiere modernizarse para enfrentar de manera efectiva los desafíos actuales del sistema de educación superior chileno.

3.2 Principales debilidades del AFI asociadas a la calidad de la educación superior

a. Limitado poder predictivo de rendimiento académico

El AFI descansa totalmente en el puntaje obtenido por los alumnos en la PSU para identificar a los mejores egresados del sistema

11 ÍNDICES 2010, Consejo Nacional de Educación. 
escolar. Si bien este criterio puede tener una justificación operativa al ser la PSU un instrumento fácilmente disponible y con cierta capacidad predictiva, su uso exclusivo implica renunciar a otros instrumentos que complementariamente podrían predecir de mejor manera el rendimiento futuro que tendrá el alumno en la educación superior. En este sentido, diversos estudios han demostrado que este tipo de prueba estandarizada, si bien ostenta un nivel predictivo significativo, también posee limitaciones. Consistentemente, la evidencia empírica sugiere que su uso debería ser complementado con otros instrumentos o indicadores que permitan identificar a los mejores alumnos con mayor precisión.

Para explicar la varianza en el rendimiento académico de los estudiantes universitarios algunos estudios se centran en el efecto de variables sociodemográficas del alumno y sus familias. Otros se centran en el impacto de variables no cognitivas, mientras que un tercer grupo lo hace en variables cognitivas o del ámbito académico. Dentro de los primeros, Betts y Morell (1999) identifican factores como el género, el nivel socioeconómico familiar y el origen étnico que explican significativamente las notas promedio de los alumnos.

Dentro de los segundos, Lotkowski, Robbins y Noeth (2004), mediante un meta-análisis de más de 100 estudios enfocados en alumnos post-secundarios de Estados Unidos, encuentran que la "confianza en el propio rendimiento académico" y la "motivación de logro" del alumno se encuentran fuertemente correlacionadas con su nivel de desempeño efectivo.

Finalmente, dentro del tercer grupo de estudios, Edge y Friedberg (1984), Cohn, et al. (2004) y Baron y Norman (1992), utilizan como variables explicativas del desempeño académico el puntaje obtenido por el estudiante en pruebas estandarizadas de ingreso a las instituciones de educación superior ${ }^{12}$, el promedio de sus notas de secundaria y su ranking de egreso de secundaria, encontrando

12 Como el SAT (Scholastic Assessment Test) y el ACT (American College Testing Program), que son pruebas estandarizadas frecuentemente utilizadas por las instituciones de educación superior de EE.UU. para seleccionar a sus alumnos. 
en especial una alta capacidad predictiva de este último. En esta misma línea, Contreras et al. (2009), al analizar el desempeño de primer año de 9.000 alumnos de cuatro universidades chilenas, también demuestran la importancia del ranking de egreso de secundaria como variable explicativa del rendimiento en la universidad. Por tanto, pareciera que esta variable incorpora aspectos no contenidos en las notas promedio de enseñanza media ni en el puntaje obtenido en la PSU, aportando, probablemente, información del alumno vinculada a su perseverancia, motivación, competitividad, técnicas de estudio y habilidad relativa.

En conclusión, a la luz de la evidencia, utilizar el puntaje en la PSU para identificar a los mejores alumnos y, por tanto, premiar su atracción por parte de las instituciones de educación superior, posee un poder predictivo limitado, que debiese ser complementado con otros indicadores. Entre dichos indicadores complementarios se destacan las notas y/o el ranking de egreso de enseñanza media del alumno, en relación con el resto de los alumnos del establecimiento, los que junto con tener un importante poder predictivo, no implican incrementar de manera significativa los costos operativos del sistema, pues al igual que la PSU, son instrumentos fácilmente disponibles.

b. Foco en la atracción de alumnos, pero no en su retención El diseño actual del AFI premia la atracción de los alumnos en el primer año, lo que es positivo, pero no premia su retención en los años posteriores, ni su titulación oportuna. Esto es un aspecto relevante de abordar debido a las altas tasas de deserción y bajas tasas de titulación oportuna existentes en el sistema terciario chileno ${ }^{13}$.

Ambos fenómenos generan costos significativos tanto para el Estado como para las familias, impactando más a aquellas de menores recursos. Adicionalmente, la evidencia muestra que los estudiantes que abandonan sus estudios acceden a menores salarios en el mercado laboral y tienen mayores riesgos de desempleo en comparación con

13 Existen diversos estudios que han investigado las causas subyacentes a la deserción estudiantil en el nivel terciario, encontrando múltiples factores explicativos. La descripción de variables y el análisis de los principales modelos de deserción se presenta en Himmel (2002). 
aquellos que completan sus programas. Así, se estima que quienes terminan sus estudios reciben un "premio" que incluso puede superar el 45\% del ingreso de quienes no logran completarlos (González y Uribe, 2002).

Los estudios disponibles indican que las tasas de deserción son bastante altas, tanto en universidades como en institutos profesionales y centros de formación técnica. En UNESCO (2005) se estima que la tasa de deserción global en educación superior en Chile es 53,7\%, lo cual contrasta fuertemente con el $31 \%$ promedio de los países miembros de la OCDE (OCDE, 2008). Esta tasa es menor en las universidades pertenecientes al CRUCH (50\%) que en las restantes (63\%). En áreas como las Humanidades y Derecho, las tasas proyectadas ascienden a niveles cercanos al $80 \%$. A su vez, en UNESCO $(2006)^{14}$ se muestra que la tasa de deserción entre el año 1 y el 4 de los alumnos de los dos quintiles de mayor ingreso es de $28 \%$, mientras que la de los alumnos de los dos quintiles de menor ingreso es de $65 \%$. Este mismo estudio estima que la tasas de titulación oportuna son reducidas, encontrando que tan solo $22 \%$ de los estudiantes logra titularse dentro del periodo oficialmente estipulado para ello y solo otro $23 \%$ de alumnos lo hace con un año de retraso.

Según el Sistema Nacional de Información de la Educación Superior (SIES) (2010), la tasa de deserción del primer año llega al 25\% en las universidades, a $42 \%$ en los institutos profesionales y a $37,5 \%$ en los centros de formación técnica. Al analizar las tasas de deserción por tipo de carrera, estas son mayores en las técnicas (41\%) que en las profesionales (28\%), mientras que por área de estudio, nuevamente Derecho aparece como aquella con mayor deserción (52\%).

Los niveles de deserción observados debiesen ser motivo de preocupación, ya que generan un costo social significativo que sería posible reducir con políticas que apoyen la retención, combinadas con mejor orientación para elegir áreas de estudio al egresar de enseñanza secundaria. En UNESCO (2005) se estima, de manera gruesa, que el costo anual de la deserción estudiantil terciaria

14 Citado en OCDE y Banco Mundial (2009). 
equivaldría al 26\% del gasto público destinado a la educación universitaria en Chile ${ }^{15}$.

En consecuencia, en el contexto del AFI, sería conveniente aprovechar su potencial generador de incentivos para que las instituciones de educación superior reduzcan sus niveles de deserción y aumenten sus tasas de titulación oportuna. En particular, se debe incentivar a las instituciones para que indaguen las variables que estarían a su alcance para reducir la deserción estudiantil, y para que consecuentemente apliquen estrategias efectivas que permitan retener a los estudiantes y velar para que la mayoría de ellos complete sus programas de estudio a tiempo.

c. A las instituciones de educación superior no se les exigen requisitos de calidad para recibir $\mathrm{AFI}$

En Chile existen dos instituciones que velan por la calidad de la educación superior: el Consejo Nacional de Educación, que se encarga de otorgar licenciamiento a nuevas universidades, institutos profesionales y centros de formación técnica mediante un proceso que evalúa el avance y concreción del proyecto educativo de cada entidad; y la Comisión Nacional de Acreditación, que verifica y promueve carreras y programas que ofrecen las instituciones de educación superior mediante el proceso de acreditación. Este último proceso es de carácter voluntario y entrega una certificación de calidad de los procesos internos de las distintas instituciones, así como de sus carreras de pregrado y programas de posgrado.

En los últimos años se han observado avances en condicionar la entrega de los distintos beneficios monetarios que entrega el Estado a los estudiantes a la acreditación de las instituciones de educación superior en que se matriculan. Ello ha ocurrido en la mayoría de las becas de arancel y mantención, así como en el Crédito con Aval del Estado. Sin embargo, percibir el AFI no exige la acreditación de la institución receptora.

15 Cálculo referencial realizado para el año 2005, considerando solo gasto público orientado a universidades. 
Esto es un hecho relevante, pues del total de los establecimientos de educación superior existentes en 2009 , solo el 38\% se encontraba acreditado (incluyendo un $77 \%$ de las universidades, un 29\% de los institutos profesionales y un $12 \%$ de los centros de formación técnica (tabla 2)). De las instituciones que percibieron recursos del AFI en 2009, el 33\% no se encontraba acreditada. Aunque dichas instituciones captaron solo el 7,3\% de los recursos del AFI, de todas maneras implica que un porcentaje de los alumnos causantes del beneficio estudia en instituciones no acreditadas $^{16}$.

Tabla 2. Porcentaje de instituciones de educación superior acreditadas, 2009

\begin{tabular}{|l|c|}
\hline Institución de educación superior & Porcentaje acreditadas del total de instituciones \\
\hline Universidades & $77 \%$ \\
CRUCH & $96 \%$ \\
Otras & $64 \%$ \\
\hline & $29 \%$ \\
\hline Centros de formación técnica & $12 \%$ \\
\hline Total & $38 \%$ \\
\hline
\end{tabular}

Fuente: elaborada sobre la base de datos de la Comisión Nacional de Acreditación y de la División de Educación Superior del Ministerio de Educación.

Dado que las instituciones de educación superior que reciben AFI no requieren cumplir ningún requisito excepto matricular a alumnos causantes de dicho aporte, aquellas que no cumplen con la acreditación podrían estar matriculando alumnos AFI exclusivamente por sus estrategias comerciales. Indicio de esto es la competencia por captar alumnos con AFI a través de beneficios económicos complementarios que las instituciones de educación superior ofrecen a los postulantes con buenos puntajes PSU, como becas, rebajas de arancel y créditos institucionales, entre otros (Rojas y Bernasconi, 2002). Esto no es problema en sí, salvo que no se exige que las instituciones que atraigan a los alumnos con AFI tengan un nivel de calidad acreditado y, por tanto, los recursos fiscales del AFI y los aportes de las propias familias pueden estar en parte siendo destinados a financiar instituciones que no proveen una educación de calidad.

16 Para efectos del presente estudio no se pudo recabar la cantidad de alumnos causantes AFI matriculados en instituciones no acreditadas. Pero dado que los alumnos de mayores puntajes PSU tienden a matricularse en instituciones acreditadas y que ellos generan un mayor monto AFI per cápita, se puede asumir que representan un porcentaje al menos igual a 7,3\%, lo que equivale a cerca de 2 mil alumnos por año. 
En este sentido, aunque los estudiantes que dan origen a AFI que se matriculan en instituciones no son la mayoría, se estima que se está desaprovechando el poder del AFI para incentivar conductas deseables en las instituciones de educación superior, como serían en este caso lograr su acreditación.

\subsection{Principales debilidades del AFI asociadas a la equidad} del sistema de educación superior

a. Sesgo en contra de los alumnos de menor nivel socioeconómico El AFI, al basar su distribución en los puntajes obtenidos por los estudiantes en la PSU, hereda el sesgo en contra de los alumnos de menor nivel socioeconómico de dicho instrumento ${ }^{17}$.

En efecto, el puntaje de la PSU se encuentra fuertemente correlacionado con una serie de factores ajenos a las habilidades cognitivas o inteligencia del postulante, como su nivel socioeconómico familiar y la educación de sus padres (Contreras et al, 2007). Esto ha llevado a diversos autores a cuestionar la pertinencia de la PSU por afectar el acceso equitativo al sistema de educación superior y, por ende, por limitar la movilidad social.

Este sesgo por factores individuales, familiares y escolares se acentúa por el dispar acceso a preuniversitarios y cursos intensivos de entrenamiento para rendir la PSU. El alto costo de este tipo de perfeccionamientos hace que no estén disponibles de manera uniforme para todos los estudiantes de educación media, a pesar del importante efecto que estos tendrían en los alumnos, incluso para los provenientes de niveles socioeconómicos bajos, como muestran Núñez y Millán (2002).

Por lo tanto, la utilización exclusiva de la PSU como indicador de la calidad de los alumnos para determinar la asignación del AFI, lleva a que la gran mayoría de los causantes del mismo sean alumnos de los quintiles de mayores ingresos. Esta situación se acentúa por la existencia de los cinco tramos de AFI que generan pagos crecientes

17 Sesgos similares existían cuando se utilizaba la PAA. Al respecto ver Contreras, Bravo y Sanhueza (2001). 
a las instituciones según los resultados en la PSU obtenidos por los causantes de AFI matriculados en ellas. Como se señaló, un alumno del tramo de mayor puntaje genera 12 veces más recursos que uno del tramo de menor puntaje. Dado que la presencia de alumnos de nivel socioeconómico alto aumenta en los tramos de mayores puntajes (gráfico 4), esto hace aún más atractivo para las instituciones captar a estos alumnos, los que a la vez son en promedio menos costosos de educar.

Esto se refleja en que los alumnos del quintil de mayores ingresos representan un 50\% de los causantes (gráfico 4) y generan más del 56\% de los recursos del AFI (tabla 3).

Gráfico 4. Distribución de alumnos causantes de AFI según quintil de ingreso per cápita del hogar*

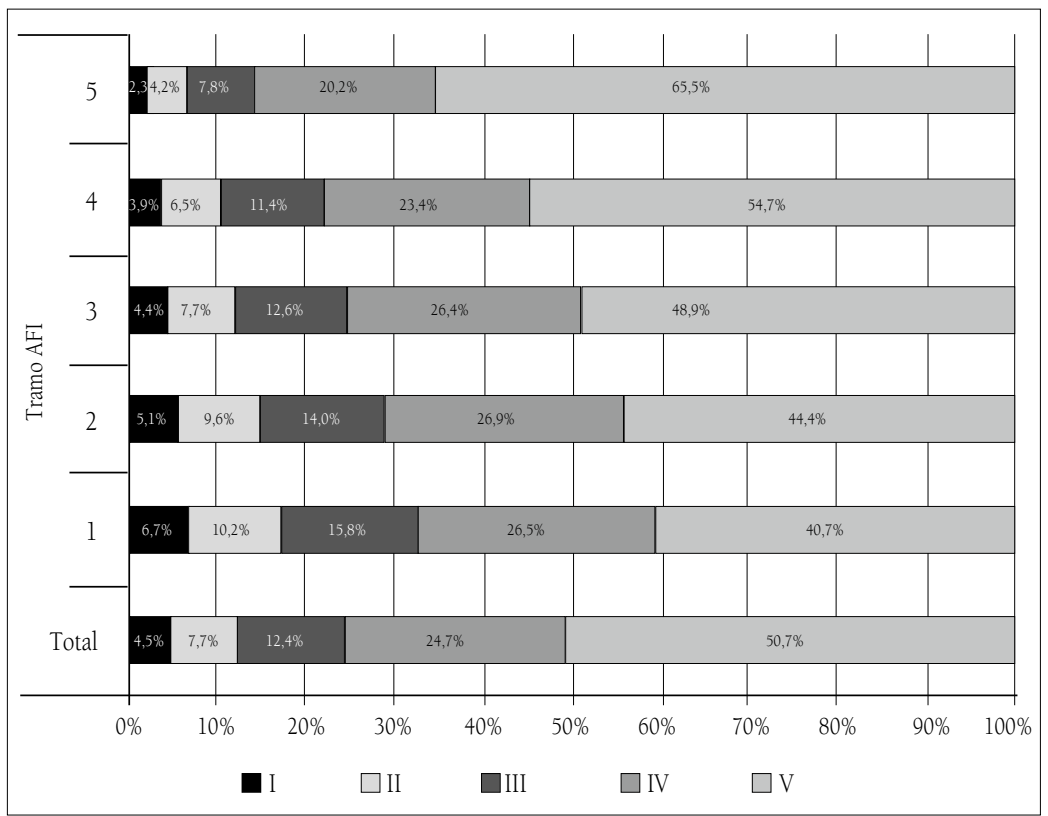

Fuente: elaborado sobre la base de PSU 2009.

* Corresponde al ingreso de los hogares declarados en el Formulario Único de Acreditación Socioeconómica (FUAS). 
Tabla 3. Distribución de los recursos del AFI según quintil de ingreso per cápita del hogar de los alumnos causantes*

\begin{tabular}{|c|c|}
\hline Quintil de ingreso per cápita & \% de recursos del AFI \\
\hline I & $3,6 \%$ \\
\hline II & $6,3 \%$ \\
\hline III & $10,7 \%$ \\
\hline IV & $23,2 \%$ \\
\hline V & $56,1 \%$ \\
\hline Total & $100 \%$ \\
\hline
\end{tabular}

Fuente: elaborado sobre la base de PSU 2009.

* Corresponde al ingreso de los hogares declarados en el Formulario Único de Acreditación Socioeconómica (FUAS).

La distribución de estudiantes causantes de AFI por nivel socioeconómico está a su vez altamente correlacionada con la distribución de causantes AFI por tipo de establecimiento de egreso de enseñanza media, pues en promedio los alumnos provenientes de establecimientos municipales obtienen peores resultados en la PSU que aquellos provenientes de establecimientos particulares subvencionados, y aun peores que sus pares egresados de colegios particulares pagados.

Esto se refleja claramente en la distribución de alumnos causantes de AFI según su establecimiento de egreso de educación media, la que se concentra en los egresados de colegios particulares pagados (gráfico 5), situación que se magnifica en términos de los recursos AFI asociados a estos alumnos por la ya señalada existencia de los cinco tramos de AFI que generan pagos crecientes a las instituciones según los resultados en la PSU obtenidos por los causantes de AFI matriculados en ellas. Así, los egresados de colegios particulares pagados generan el 45,0\% de los recursos del AFI, porcentaje superior al ya alto $38,6 \%$ que representan en cantidad de alumnos causantes de AFI (tabla 4). 
Gráfico 5. Distribución de alumnos causantes de AFI según establecimiento de egreso de educación media

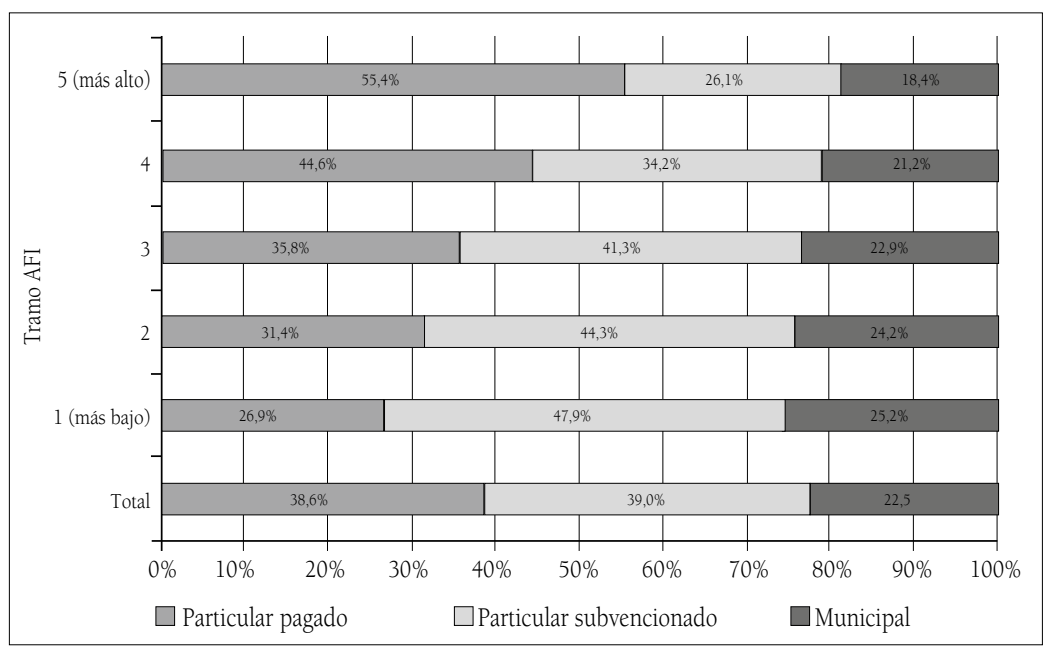

Fuente: elaborado sobre la base de PSU 2009.

Tabla 4. Distribución de alumnos según tipo de establecimiento de egreso de educación media

\begin{tabular}{|l|c|c|c|c|}
\hline Dependencia & $\begin{array}{c}\text { Matrícula } 4^{\circ} \\
\text { medio }\end{array}$ & $\begin{array}{c}\text { Recién egresados } \\
\text { inscritos PSU }\end{array}$ & $\begin{array}{c}\text { \% Causante AFI } \\
\text { del total }\end{array}$ & $\begin{array}{c}\text { \% Estimado de } \\
\text { recursos AFI del } \\
\text { total }\end{array}$ \\
\hline Particular pagado & $8,6 \%$ & $12,1 \%$ & $38,6 \%$ & $45,0 \%$ \\
\hline Particular subvencionado & $47,0 \%$ & $47,5 \%$ & $39,0 \%$ & $34,0 \%$ \\
\hline Municipal & $44,3 \%$ & $39,9 \%$ & $22,5 \%$ & $20,9 \%$ \\
\hline Total & $100 \%$ & $100 \%$ & $100 \%$ & $100 \%$ \\
\hline
\end{tabular}

Fuente: elaborado sobre la base de PSU 2009 y Estadísticas de la Educación 2006-2007, Ministerio de Educación.

En conclusión, la distribución de recursos del AFI, al determinarse exclusivamente según los puntajes de la PSU, hace que en la práctica se acentúe desproporcionadamente la competencia entre las instituciones de educación superior por captar a estudiantes del quinto quintil egresados de colegios particulares pagados, pues con alta probabilidad serán causantes de AFI.

b. Pagos del AFI no están asociados al costo de educar al alumno La evidencia indica que el capital humano acumulado de un alumno está fuertemente afectado por las condiciones de su hogar, razón 
por la cual los estudiantes de bajo nivel socioeconómico presentan desventajas de origen. El gasto que es necesario realizar en términos de horas de educación, de material educativo y de reforzamiento, entre otros, para llevar a un estudiante a un nivel determinado de conocimientos, es función del capital humano que este trae del hogar, y dicho costo aumenta cuanto menor es su capital humano acumulado. En consecuencia, el costo de educar a un alumno está inversamente correlacionado con su nivel socioeconómico (Sapelli, 2006).

La introducción de la Subvención de Educacional Preferencial (SEP) en la educación general es la primera experiencia en Chile que reconoce explícitamente la existencia de diferencias de origen que inciden en el costo de proveer educación. Para compensar estas diferencias, la SEP entrega una mayor subvención por alumno a aquellos considerados vulnerables (Darville y Rodríguez, 2007).

Un concepto similar no se ha evaluado para la educación terciaria. Es así como el AFI no se hace cargo de este diferencial en el costo de educar. Por el contrario, entrega más recursos a las instituciones de educación superior que captan a los alumnos con mejores puntajes en la PSU, quienes a su vez en promedio presentan mayor nivel socioeconómico y, por tanto, son los menos costosos de educar. Por ende, el AFI no solo no se asocia al costo de educar a los alumnos, sino que tiende a entregar mayores recursos en función de matricular a aquellos que son menos costosos de educar.

En conclusión, el diseño actual del AFI crea un incentivo adicional a las instituciones de educación superior por atraer a los alumnos de mayor nivel socioeconómico, ya que entrega mayores recursos por educar a aquellos de mayor capital humano inicial que, consistentemente, son los menos costosos de educar. Esto acentúa la competencia entre las instituciones de educación superior por atraer y matricular a este tipo de alumnos, lo que en cierto grado tiende a perpetuar la segregación del sistema escolar hacia el sistema terciario.

c. Limitaciones de la PSU para educación media técnico profesional Si bien el AFI se creó como un instrumento para incentivar la atracción de los mejores estudiantes de enseñanza media a las instituciones 
de educación superior, sin distinguir el tipo de enseñanza media, en la práctica, como utiliza la PSU como indicador de calidad del estudiante, tiene un sesgo en contra de los alumnos provenientes de liceos técnico-profesionales (EMTP).

La causa de este sesgo es que la PSU fue creada y es aplicada por las universidades del CRUCH con el fin de constituir el principal instrumento de selección de los alumnos que postulan a ellas. Por lo tanto, posee una orientación académica, considerando los contenidos del Plan de Formación General de Lenguaje y Comunicación, de Matemática, de Historia y Ciencias Sociales, y de Ciencias.

En este sentido, los estudiantes de EMTP tienen una desventaja, por cuanto reciben un menor grado de formación general después de segundo medio. De hecho, según se señala en el Informe Final de la Comisión de Formación Técnica Profesional, convocada en 2008 por el Ministerio de Educación, "los alumnos de la modalidad técnico-profesional (TP) están significativamente menos expuestos a la formación general en comparación a los alumnos de la modalidad científico-humanista $(\mathrm{CH})$. Mientras un alumno de la modalidad $\mathrm{CH}$ cuenta con 27 horas semanales de formación general, uno de la modalidad TP tiene solo 12". El resultado de esto es que consistentemente los alumnos de EMTP obtienen en promedio puntajes más bajos en la PSU que sus pares que estudiaron en EMCH. En efecto, en los procesos del periodo 2008-2010 cerca del 68\% de los alumnos de EMCH que rindieron la PSU obtuvo un puntaje igual o superior a 475, mientras que solo alrededor de un 37\% de los alumnos de EMTP que rindieron la PSU obtuvo un rendimiento comparable (tabla 5) ${ }^{18}$.

Adicionalmente, se verifica que el porcentaje de estudiantes de EMTP que rinde la PSU es muy inferior al de los estudiantes de $\mathrm{EMCH}$. Así, de los 89.000 estudiantes que se encontraban en el último año de EMTP en 2004, aproximadamente solo 27.000 (el 30\%), rindieron la PSU para matricularse en el proceso de admisión 2005. En contraste, de los 105.000 estudiantes que se encontraban en el

18 El puntaje PSU de 475 (promedio de Lenguaje y Matemática) corresponde al mínimo requerido para que estudiantes universitarios accedan al Crédito con Aval de Estado para educación superior, por lo que resulta útil como referencia analítica. 
último año de EMCH en 2004, cerca de 95.000 (el 90\%), rindieron la PSU para participar del proceso de admisión $2005^{19}$. Esto podría explicarse, entre otras razones, debido a que las instituciones técnicoprofesionales de nivel superior por lo general no exigen la PSU, o si la exigen, no toman en cuenta su puntaje para efectos del proceso de admisión, sino que solo el hecho de haberla rendido (OCDE, 2009). Por lo tanto, los incentivos a rendir la PSU y, más aún, para obtener un buen rendimiento en ella, son bajos, incluso para los mejores alumnos de EMTP que deseen estudiar una carrera técnico-profesional.

Tabla 5. Estudiantes con puntaje PSU promedio igual o mayor a 475, por tipo de enseñanza media 2008-2010

\begin{tabular}{|l|r|r|c|r|r|r|r|r|r|}
\hline Rama & \multicolumn{3}{|c|}{2008} & \multicolumn{3}{c|}{2009} & \multicolumn{3}{c|}{2010} \\
\hline & Rinden & \multicolumn{2}{c|}{$>=475$} & Rinden & \multicolumn{2}{c|}{$>=475$} & Rinden & \multicolumn{1}{c|}{$>=475$} \\
\hline & & \multicolumn{1}{c|}{$\mathrm{N}$} & $\%$ & & \multicolumn{1}{c|}{$\mathrm{N}$} & \multicolumn{1}{c|}{$\%$} & $\mathrm{~N}$ & $\%$ \\
\hline Cientif.-humanista & 154.140 & 104.492 & 67,79 & 169.562 & 115.140 & 67,9 & 174 & 118.293 & 67,88 \\
\hline Téc.-profesional & 61.374 & 22.609 & 36,84 & 71.120 & 26.528 & 37,3 & 75.301 & 28.493 & 37,84 \\
\hline Validaciones & 1.272 & 571 & 44,89 & 1.327 & 659 & 49,3 & 1.903 & 973 & 51,13 \\
\hline Reconocimientos & 82 & 73 & 89,02 & 109 & 96 & 88,07 & 121 & 106 & 80,92 \\
\hline Sin información & 13 & 6 & 46,15 & 19 & 7 & 36,84 & 3 & - & 0 \\
\hline Total & 216.881 & 127.751 & 58,9 & 242.000 & 142.423 & 58,83 & 251.617 & 147.865 & 58,77 \\
\hline
\end{tabular}

Fuente: DEMRE.

En síntesis, debido a que los alumnos de EMTP tienen un currículum distinto a los de EMCH, sumado a que la PSU se centra más en medir los aprendizajes de estos últimos, implica que el puntaje en esta prueba no resulta un buen predictor del desempeño académico futuro de este tipo de estudiantes. Además, considerar el puntaje PSU para asignar los recursos del AFI de alguna manera castiga la decisión coherente de un alumno que sigue EMTP y luego continúa sus estudios en una carrera superior técnica, ya que estas instituciones tienden a no exigirla en sus procesos de admisión. Por lo tanto, implícitamente, al basarse exclusivamente en los puntajes de la PSU para su distribución, el AFI limita su poder de crear incentivos virtuosos en la enseñanza técnico-profesional.

19 Mayores antecedentes en Valdivieso et al. (2006). Es importante tener en cuenta que estos datos son aproximados, ya que parte de la cohorte matriculada en $4^{\circ}$ medio no logra graduarse y, por ende, no rinde la PSU. Asimismo, parte de las personas que rinden la PSU en un año en particular no corresponde a recién egresados. 


\section{Propuesta para el perfeccionamiento del AFI}

\subsection{Objetivos del Aporte Fiscal Indirecto}

Como se ha señalado, el AFI actual en sus orígenes tenía por finalidad incentivar la calidad de la educación por medio de la competencia, por parte de las universidades, de captar a los mejores alumnos. Sin embargo, como se discutió en la sección 3 del presente trabajo, la forma de determinación actual de lo que se define por alumno de calidad es discutible, y se encuentra fuertemente correlacionada con su nivel socioeconómico y establecimiento de egreso de enseñanza media, privilegiando a aquellos de nivel socioeconómico alto egresados de establecimientos particulares pagados.

Por ello, se plantea reformular el objetivo del AFI, para que incentive la calidad y vele por la eficiencia y, a la vez, mejore la equidad de acceso y retención del sistema de educación superior.

Para cumplir este nuevo objetivo se propone el rediseño del AFI que se describe en los puntos siguientes de esta sección.

\subsection{Diseño conceptual}

a. Mayor peso relativo del AFI dentro del sistema de financiamiento público de la educación superior

Para que el AFI genere reales incentivos en las instituciones de educación superior, es indispensable que aumente su peso relativo dentro de las fuentes de apoyo fiscal para la educación superior en Chile. El AFI hoy representa alrededor de 11\% del aporte fiscal para las instituciones y solo un 3,5\% del aporte fiscal total para educación superior. En contraste, el AFD, que se rige mayoritariamente por criterios históricos, representa un $72 \%$ y un $25 \%$, respectivamente.

Para que el AFI no pierda peso relativo en el tiempo, se propone que la cantidad de alumnos causantes AFI se defina como un porcentaje meta de la matrícula de primer año del periodo anterior (no como una cantidad fija, como es en la actualidad) y que en términos de recursos nunca sea inferior a un porcentaje meta respecto del financiamiento público total para las instituciones de educación superior. 
El mayor peso relativo y cobertura del AFI fortalecería los incentivos que genera, ampliando la cantidad de instituciones que se esforzaría por captar estos recursos, a diferencia del esquema actual en que la competencia efectiva se concentra en unas pocas instituciones tradicionales.

La mayor importancia relativa del AFI dentro de los instrumentos existentes de financiamiento público de la educación superior se puede lograr asignándole presupuesto adicional, sin que implique disminuir los recursos en términos absolutos del resto de los instrumentos. Aumentar los recursos fiscales para educación superior en términos absolutos iría en línea con acercarse a los parámetros de los países de la $\mathrm{OCDE}^{20}$. No obstante, si se tiene en cuenta que los recursos fiscales son escasos, resulta pertinente identificar si existe algún instrumento que deba reducir su peso incluso en términos absolutos, para incrementar el peso del AFI. En el presente trabajo no se ha realizado una evaluación en profundidad del resto de los instrumentos de financiamiento de la educación superior como para afirmar, de manera concluyente, cuál debe ser reducido para reasignar recursos en favor del AFI. Sin embargo, siguiendo los principios orientadores para la inversión pública en educación superior discutidos en Rodríguez et al. (2010) y en Flores et al. (2010), los candidatos debiesen ser aquellos instrumentos que se rigen por criterios históricos y que en sí mismos no fomentan la calidad ni la competencia, como por ejemplo el Aporte Fiscal Directo (AFD).

b. Uso de indicadores complementarios para la identificación del potencial académico de los estudiantes

El AFI debe mejorar los indicadores utilizados para definir e identificar quienes son considerados alumnos con alto potencial académico. Además, debe integrar de una manera pertinente y efectiva a las instituciones de educación superior que imparten carreras técnicoprofesionales.

20 Mientras el gasto público en educación superior en Chile representa un 0,3\% del PIB, en los países de la OCDE este alcanza un 1,3\% del PIB en promedio (OCDE, 2009). 
Con este objetivo, para compensar los sesgos de la PSU en contra de los estudiantes de menor nivel socioeconómico que postulan a universidades e institutos profesionales y de aquellos que se matriculan en centros de formación técnica, se propone, para los primeros, crear un algoritmo que considere la PSU y el ranking relativo de egreso de enseñanza media del alumno, en relación con el resto de los alumnos del establecimiento ${ }^{21} \mathrm{y}$, para los segundos, sustituir la PSU por un algoritmo que considere el promedio de notas y el ranking relativo de egreso de enseñanza media ${ }^{22}$.

Complementariamente, dado que bajo esta propuesta la construcción de puntajes AFI sería diferenciada entre los alumnos que eligen carreras técnicas de quienes eligen carreras profesionales, resulta pertinente que no compitan entre sí como causantes de recursos. En la práctica, esto implica tener un AFI para carreras técnicas y otro para carreras profesionales.

\section{c. Incentivo a la retención y titulación oportuna}

Para incentivar la calidad de la educación superior, no basta atraer buenos estudiantes cada año si no se garantiza que un porcentaje significativo de ellos se mantenga estudiando y se titule oportunamente. Por ello, se propone un esquema de pagos a las instituciones de educación superior que las incentive a aumentar la retención y titulación oportuna de sus alumnos, por medio de implementar actividades orientadas a mejorar sus capacidades de orientación vocacional, reforzamiento de contenidos para los alumnos (como comprensión lectora, escritura y matemáticas), métodos de

21 En un principio se consideró incluir también el promedio de notas de enseñanza media, recomendado en la literatura internacional como buen predictor académico. Sin embargo, se descartó pues para el caso chileno dicha variable genera sesgos a favor de los alumnos de mayor ingreso, lo que representa una característica no deseada. En efecto, utilizando la base de datos del proceso PSU 2009, el promedio de notas presentan un coeficiente de correlación de 0,15 con respecto al quintil de ingresos del hogar. Así por ejemplo, alumnos pertenecientes al primer quintil presentan un puntaje por notas de enseñanza media igual a 494 , mientras que los del quinto quintil presentan un puntaje de 564. El ranking de egreso no presenta este problema, pues su correlación con el quintil de ingresos es de solo 0,02.

22 En este caso se propone no considerar la PSU, por no ser una prueba de selección de carreras técnicas. De existir una prueba especial con esas características podría ser pertinente considerarla. A su vez, se propone incluir el promedio de notas de enseñanza media, a pesar de la ya señalada alta correlación con nivel socioeconómico, para no dejar todo el peso relativo en una sola variable. 
estudios y apoyo psicosocial. Si bien en teoría estas medidas debiesen orientarse a los alumnos AFI, en la práctica se esperaría que beneficien a la totalidad de los estudiantes. Incluso, en el mediano y largo plazo, podría ocurrir que las buenas prácticas fueran adoptadas por instituciones no beneficiarias del AFI.

Para ello, se propone que en vez de entregar el monto asociado al AFI por cada estudiante en una sola cuota para el primer año, como es en la actualidad, se introduzca un incentivo a la retención de alumnos vulnerables y a su graduación en un tiempo prudente, entregándose el AFI en cuotas crecientes a lo largo de la trayectoria del estudiante durante su carrera, donde la última cuota se entregue contra la finalización oportuna de los estudios ${ }^{23}$.

\section{d. Ajuste del monto de AFI por estudiante según su nivel socioeconómico}

Aun cuando las modificaciones propuestas al instrumento que determina quienes son los alumnos causantes de AFI corrige el sesgo negativo que actualmente existe contra los alumnos de menor nivel socioeconómico, de todas maneras puede persistir el incentivo de las instituciones de educación superior a la selección de alumnos, debido a que aquellos de menores ingresos son relativamente más costosos de educar. Este fenómeno se vería reforzado por la propuesta anterior orientada a incentivar la retención y titulación oportuna de los alumnos.

Por lo tanto, para revertir esta situación, hacer más factible los incentivos a la retención y titulación oportuna, y reconocer que el proceso educativo de los alumnos de menor nivel socioeconómico puede resultar más costoso para las instituciones, se propone que los pagos del AFI por estudiante sean inversamente proporcionales a su nivel socioeconómico. Esto reemplazaría el esquema actual en que los pagos son proporcionales al puntaje PSU. Dado que en el esquema propuesto los alumnos AFI serían los mejores alumnos que ingresan al sistema terciario, no consideramos prioritario generar una mayor segmentación dentro de ese subconjunto según capacidad

23 El mecanismo de pago en cuotas deberá velar para que las instituciones no generen trabas excesivas a aquellos alumnos causantes de AFI que desean cambiarse de carrera. 
académica, pero sí reconocer que algunos de ellos son más costosos de educar ${ }^{24}$.

e. Garantizar la calidad educativa de las instituciones receptoras del AFI

Aprovechando el fuerte poder de incentivo que tendría un AFI con un alto peso relativo en el sistema de financiamiento público para la educación superior, se propone que se utilice para incentivar la acreditación de las instituciones de educación superior. Por lo tanto, se sugiere que la recepción de AFI esté acotada exclusivamente a las instituciones acreditadas.

\section{Conclusiones y lineamientos de política}

En la última década el Estado de Chile aumentó fuertemente el financiamiento para educación superior, lo que se ha justificado por las altas externalidades positivas que ella tiene para el país. En este contexto, el mercado de la educación superior ha sido muy dinámico $y$, por ende, hoy resulta pertinente revisar y evaluar las políticas e instrumentos existentes para verificar si efectivamente cumplen sus objetivos y si se encuentran alineados con las necesidades actuales de política pública.

Teniendo en mente que es necesario desarrollar un sistema de financiamiento integrado y coherente, que contribuya a aumentar la equidad, calidad y eficiencia de la educación superior en Chile, el eje de este trabajo ha sido realizar un ejercicio de revisión y plantear una propuesta de modernización de un instrumento, el AFI, el

24 Si se quisiese premiar más a las instituciones que captan a los alumnos con mayor potencial académico dentro de los causantes AFI (determinados según puntaje PSU y ranking de egreso en el caso de los egresados de enseñanza media científico-humanista; y por el promedio de notas y ranking de egreso en el caso de los egresados de enseñanza media técnico-profesional), se podría considerar ajustar el monto del AFI en función de dichos algoritmos. Ello podría hacerse de manera complementaria al ajuste propuesto por el nivel socioeconómico de los alumnos causantes AFI. Considerando que ambos ajustes en conjunto pueden complejizar el instrumento a un punto tal que para las instituciones de educación superior no sea evidente identificar a los causantes AFI que generan mayor cantidad de recursos (y por tanto perdiéndose el poder de generar incentivos del AFI a este respecto), es que se propone privilegiar realizar solo el ajuste por nivel socioeconómico, por considerarse prioritario incorporar este elemento de mayor equidad. 
que consideramos permite dar pasos para orientar al sistema en la dirección deseada.

Al respecto, se concluye que el AFI genera incentivos para competir por los alumnos que dan derecho a percibir sus recursos, por lo que posee un alto potencial para orientarlos a mejorar la calidad de la educación terciaria de pregrado. Sin embargo, su diseño actual tiene varias debilidades que no le permiten aprovechar todo su potencial: no identifica adecuadamente a los mejores alumnos, no se adapta a la trayectoria de los estudiantes de carreras técnicas, no incentiva la retención ni titulación oportuna, no exige acreditación de las instituciones receptoras y tiene sesgos en contra de los alumnos de menor nivel socioeconómico. Resultado de esto es que los alumnos causantes del AFI son en su mayoría alumnos de ingresos altos y provenientes de establecimientos educacionales particulares.

Además, el poder de los incentivos del AFI ha caído en los últimos años junto con la disminución de su peso, tanto absoluto como relativo. Así por ejemplo, mientras en 1990 representaba el $24 \%$ del aporte fiscal para las instituciones de educación superior, en 2009 representó solo el 11\%.

A la luz de este diagnóstico, en el presente trabajo se ha planteado una propuesta de reforma del AFI orientada a aprovechar su capacidad generadora de incentivos para mejorar la calidad, eficiencia y equidad de acceso y retención de la educación superior de pregrado.

Para ello, se plantea que las instituciones beneficiarias del AFI deban necesariamente estar acreditadas; que la selección de alumnos diferencie entre carreras profesionales y técnicas; que el instrumento de identificación de los alumnos causantes de AFI considere no solo la PSU, sino también el promedio de notas (para las carreras técnicas de menos de tres años) y el ranking de egreso de enseñanza media; que el valor AFI por alumno esté determinado por su nivel socioeconómico, siendo mayor para aquellos de menores ingresos por el mayor costo que implica educarlos; y que la forma de pago del AFI sea en múltiples cuotas, de valor ascendente, generando los incentivos a la retención y a la titulación oportuna de los alumnos. 
Un elemento fundamental de la propuesta es que para que el AFI efectivamente logre los efectos deseados, debe aumentar su participación relativa dentro del total de financiamiento para educación superior (a alrededor de un 25\%) y debe aumentar el número de estudiantes causantes (cercano al 15\% de los alumnos de primer año). Al fijarse su participación relativa en términos de porcentaje de recursos y de alumnos, se evita repetir el problema de pérdida de peso relativo $y$, por ende, de fuerza de sus incentivos, lo que ha ocurrido con el instrumento desde su creación en la década de los 80.

Cabe advertir que las modificaciones propuestas implican una distribución distinta de los recursos AFI respecto de su distribución actual entre instituciones. En este sentido, en un análisis estático puede haber instituciones "ganadoras" y otras "perdedoras". Para efectos de una transición gradual que no afecte de manera brusca su planificación financiera, puede ser conveniente contemplar un fondo compensatorio que asegure una cota a la eventual pérdida de recursos respecto del AFI actual que pudieran sufrir instituciones específicas. Con todo, una compensación de este tipo debe ser solo por algunos años (no más de cinco), pues por definición las modificaciones propuestas lo que buscan es incentivar cambios en la conducta de las instituciones, las que deberán adaptarse a los nuevos incentivos para poder optar a los recursos del AFI.

La investigadora Daniela Sugg es becaria de doctorado de la Comisión Nacional de Investigación Científica y Tecnológica (CONICYT). Santiago, Chile.

\section{Referencias bibliográficas}

Baron, J. y Norman, M. (1992) SATs, Achievement Tests, and High-School Class Rank as Predictors of College Performance. Educational and Psychological Measurement, 52.

Betts, J. y Morell, D. (1999) The Determinants of Undergraduate Grade Point Average: The Relative Importance of Family Background, High School Resources, and Peer Group Effects. The Journal of Human Resources, 34 (2).

Braxton, J.; Johnson, R. y Shaw-Sullivan, A. (1997) Appraising Tinto's Theory of College Student Departure. En J. C. Smart (Ed.), Higher Education: Handbook of Theory and Research. Agathon Press. 
Brunner, J.J. (2005) Hacia una nueva politica de educación superior, en Foco $n^{\circ} 45$, Corporación Expansiva, Chile.

Cohn, E.; Cohn, S.; Balch, D. y Bradley, J. (2004) Determinants of Undergraduate GPAs: SAT scores, High-School GPA and High-School Rank. Economics of Education Review, 23.

Contreras, D.; Gallegos, S. y Meneses, F. (2009) Desempeño universitario: ¿importa la habilidad relativa?. Revista Calidad en la Educación n 30 , Consejo Superior de Educación, Chile.

Contreras, D.; Bravo D. y Sanhueza, C. (2001) PAA: ¿Una prueba de inteligencia?, Perspectivas en Política, Economía y Gestión, 4.

Contreras, D. y Macías, M. (2002) Desigualdad Educacional en Chile: Geografía y Dependencia. Cuadernos de Economía, 39 (118).

Contreras, M.; Corbalán P. y Redondo J. (2007) Cuando la suerte está echada: estudio cuantitativo de los factores asociados al rendimiento en la PSU. Facultad de Ciencias Sociales, Universidad de Chile.

Darville, P. y Rodríguez, J. (2007) Institucionalidad, financiamiento y rendición de cuentas en educación. Estudios de Finanzas Públicas n 10, Dirección de Presupuestos, Chile.

Edge, O. y Friedberg, S. (1984) Factors Effecting Achievement in the First Course in Calculus. Journal of Experimental Education, 52(3).

Flores, L.; Hernández, T.; Rodríguez, J. y Sugg, D. (2010) ¿Cómo se invierten los recursos públicos en educación superior en Chile? En Foco n ${ }^{\circ} 151$, Expansiva.

González, L. y Uribe, D. (2002) Estimaciones sobre repitencia y deserción en la educación superior chilena: consideraciones sobre sus implicancias. Revista Calidad en la Educación, n 17, Consejo Superior de Educación, Chile.

Himmel, E. (2002) Modelos de análisis de la deserción estudiantil en la educación superior. Revista Calidad en la Educación n 17, Consejo Superior de Educación. Santiago de Chile.

Lotkowski, V.; Robbins, S. y Noeth, R. (2004) The Role of Academic and NonAcademic Factors in Improving College Retention. ACT Policy Report.

MINEDUC (2009) Estadísticas de la educación 2006-2007. Ministerio de Educación, Chile.

Núñez, J. y Millán, I. (2002) ¿Pueden mejorar su PAA los alumnos de escasos recursos? Cuadernos de Economía, 29 (116).

OECD (2008) Education at a Glance. OECD Publishing, París. 
OECD (2009) Education at a Glance. OECD Publishing, París.

OCDE y Banco Mundial (2009) La educación superior en Chile. Revisión de Politicas Nacionales de Educación, París.

Rodríguez, J.; Flores, L.; Sugg, D. y Hernández, T. (2010) Inversión pública en educación superior en Chile: avances 2006-2010 y desafios. Estudios de Finanzas Públicas n 16, Dirección de Presupuestos, Chile.

Rojas, F. y Bernasconi A. (2002) AFI: un aporte a la calidad. Revista Calidad en la Educación n 17, Consejo Superior de Educación. Santiago de Chile.

Sapelli, C. (2006) Desafios del sistema educativo: la subvención preferencial. Instituto de Economía, Pontificia Universidad Católica de Chile, Año $1, n^{\circ} 1$.

Sistema Nacional de Información de la Educación Superior, SIES. (2010) Retención de primer año en el pregrado: descripción y análisis de la cohorte de ingreso 2007. Ministerio de Educación, Chile.

Tinto, V. (1975) Dropout in Higher Education: a Theoretical Synthesis of Recent Research. Review of Educational Research, 45.

UNESCO (2005) Estudio sobre la repitencia y deserción en la educación superior chilena. Programa Observatorio de la Educación Superior en América Latina y el Caribe, Instituto Internacional para la Educación Superior en América Latina y el Caribe.

Valdivieso, P.; Antivilo, A. y Barrios, R. (2006) Caracterización educacional y sociodemográfica de los estudiantes que rinden la PSU, postulan y se matriculan en las universidades reunidas en el Consejo de Rectores. Revista Calidad en la Educación n 24, Consejo Superior de Educación, Chile.

Recibido: 30/09/2011

Aceptado: 14/11/2011 


\section{Anexo}

Simulación de una propuesta específica de modificación del AFI

En este anexo, a modo ilustrativo, se simulan los efectos de una propuesta específica de modificación del AFI, que cumple con el diseño conceptual recomendado. En particular:

Para que el AFI tenga un peso relativo significativo, se propone que tenga una cobertura de al menos un 15\% de los matriculados en primer año el año anterior en universidades, Institutos Profesionales y Centros de Formación Técnica. En base a la matrícula 2009, los causantes AFI ascenderían a 39.765 alumnos, lo que significa un aumento de $45 \%$ respecto a la cantidad actual. De ellos, 5.237 cupos se destinan para alumnos que se matriculen en carreras técnicas de tres o menos años de duración.

Complementariamente, también para asegurar que el AFI tenga una alta importancia relativa como fuente de financiamiento, se propone que sus recursos representen al menos el 25\% de los recursos fiscales destinados a las instituciones de educación superior. Considerando el presupuesto para 2010, esto equivale a 50.881 millones de pesos (casi un $130 \%$ superior al presupuesto efectivo para 2010).

Para el puntaje AFI de aquellos que se matriculen en universidades o Institutos Profesionales, se propone un algoritmo que pondere en partes iguales el puntaje promedio PSU y el ranking de egreso de enseñanza media dentro de cada establecimiento. Para aquellos que se matriculen en Centros de Formación Técnica, se propone un algoritmo que pondere en partes iguales el promedio de notas de enseñanza media y el ranking de egreso de enseñanza media dentro de cada establecimiento.

El puntaje que se asocia al promedio de notas de enseñanza media corresponde al que se obtiene de la escala de transformación creada por el Departamento de Evaluación, Medición y Registro Educacional de la Universidad de Chile. El puntaje que se obtiene del ranking de egreso se estima mediante una ecuación simplificada, donde el puntaje ranking 
es igual a 826 x ranking, que hace relación al puntaje máximo a obtener si se tiene un promedio de notas igual 7 en $\mathrm{EMCH}$ diurno ${ }^{25}$.

Se propone un nuevo esquema de pagos por alumno en función de su condición socioeconómica. En particular, se simulan tres tramos de ingreso, que representan a los alumnos de menor nivel socioeconómico (quintiles I y II) con ponderación 6 , a los de nivel medio (quintiles III y IV) con ponderación 3, y de nivel alto (quintil V) con ponderación 1 , como se detalla en la Tabla $6^{26}$.

Tabla 6. Estructura propuesta del pago AFI por alumno, según quintiles de ingreso per cápita del hogar

\begin{tabular}{|c|c|c|c|}
\hline Quintiles & Coeficiente & Pago por alumno (pesos) & Número de alumnos por tramo \\
\hline I y II & 6 & 1.861 .483 & 13.043 \\
\hline III y IV & 3 & 930.742 & 22.878 \\
\hline V & 1 & 310.247 & 17.110 \\
\hline Total & & & 53.020 \\
\hline
\end{tabular}

Fuente: elaboración propia.

Se propone que los pagos del AFI se hagan en varias cuotas condicionadas a que el alumno siga matriculado en la misma carrera e institución por la que generó el AFI el primer año. Estas cuotas son diferenciadas según la duración de la carrera cursada. Para carreras con una duración mayor a tres años (carreras profesionales), se propone que las instituciones de educación superior reciban el monto del AFI correspondiente al alumno en cuatro cuotas ( $25 \%$ el primer año, $30 \%$ el segundo, $35 \%$ el último y $10 \%$ en caso de que el alumno se titule oportunamente ${ }^{27}$ ). Para carreras con una duración menor o igual a tres años (carreras técnicas), se propone que las instituciones de educación superior reciban el monto del AFI correspondiente al alumno en tres cuotas (40\% el primer año, $50 \%$ el segundo y un $10 \%$

25 Esta ecuación se eligió por simplicidad, pero podría optarse por otra, que por ejemplo, que premie de forma creciente el ranking de egreso.

26 Los montos están calculados de manera consistente con que el número de beneficiarios de AFI sea el $15 \%$ de la matrícula de primer año del año anterior, y con que los recursos totales sean equivalentes al $25 \%$ del presupuesto de aporte público a las instituciones de educación superior en 2010.

27 Se debe determinar cuáles son los tiempos de titulación oportuna para cada carrera de educación superior, considerando un margen prudente respecto del plazo teórico (por ejemplo, 1 semestre adicional). 
en caso de que se titule oportunamente). Los gráficos 6 y 7 muestran estos perfiles de pago.

Gráfico 6. Perfil de pagos del AFI a institución de educación superior por alumno matriculado en carrera de más de tres años de duración

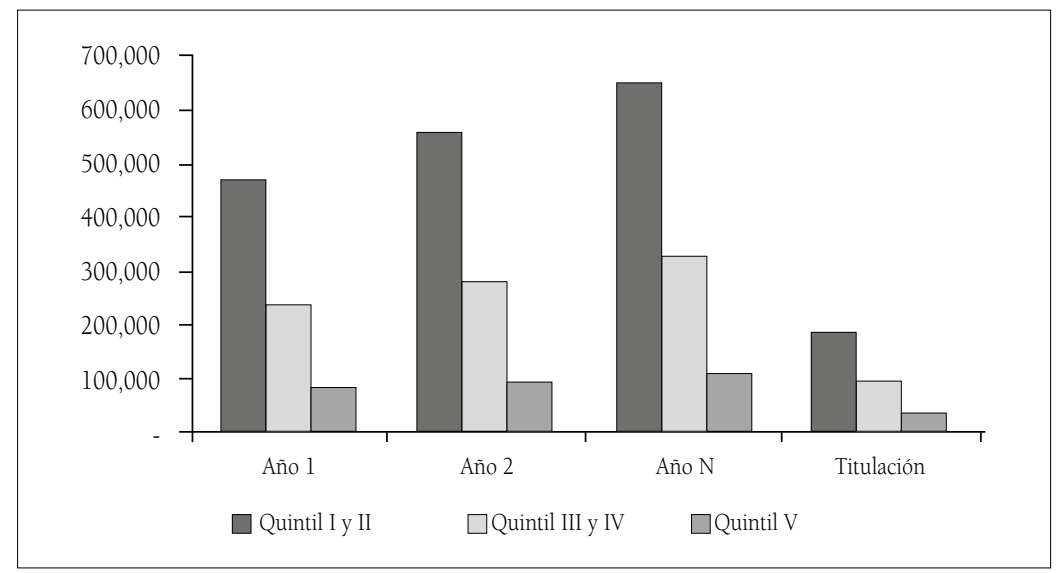

Fuente: elaboración propia.

Gráfico 7. Perfil de pagos del AFI a institución de educación superior por alumno matriculado en carrera de tres o menos años de duración

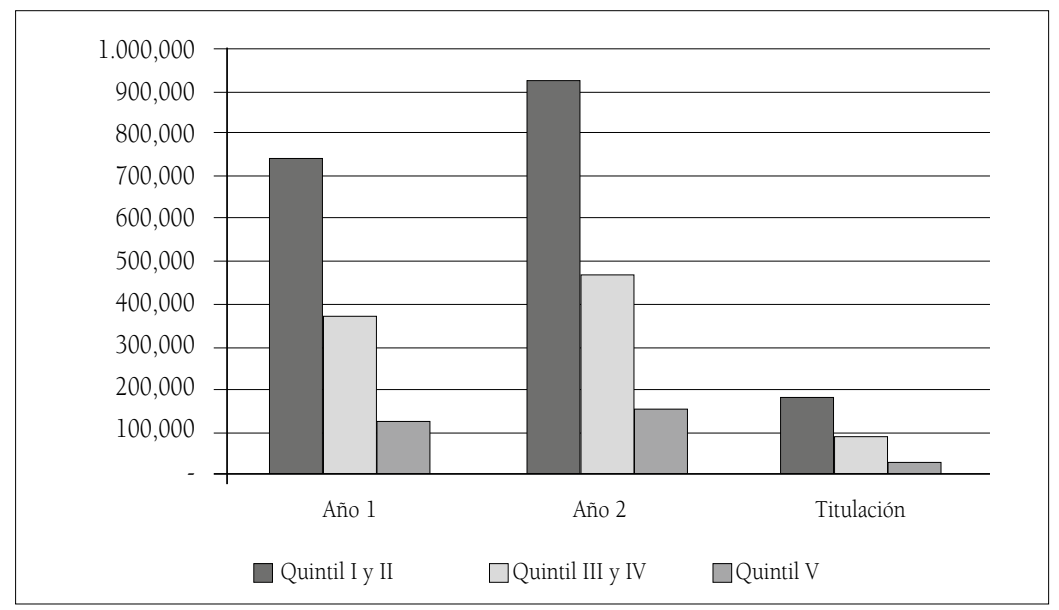

Fuente: elaboración propia.

Un primer resultado de estas propuestas es una mejora esperada en la capacidad para identificar a los estudiantes con mejor potencial académico. De la simulación, considerando la eventual entrega de AFI a 
27.500 alumnos, se identifica que el último estudiante elegible tendría 550 puntos promedio en la PSU, un promedio de notas de enseñanza media de 6,3 y se ubicaría en el 7\% mejor de su establecimiento educacional según el ranking de egreso de enseñanza media. Con el algoritmo actual para la distribución del AFI, si bien el último estudiante elegible tendría un mayor puntaje promedio en la PSU (630 puntos), tendría un peor promedio de notas de enseñanza media $(5,4)$ y un peor ranking de egreso de enseñanza media (pertenecería al 35\% mejor de su establecimiento educacional).

Un segundo resultado es que el cambio del algoritmo de cálculo para determinar a los causantes AFI genera una distribución más equitativa del mismo según nivel socioeconómico familiar, en comparación con la situación actual (gráfico 8 versus gráfico 4). Así por ejemplo, los alumnos del quinto quintil pasan de representar un $50,7 \%$ de los causantes potenciales a representar un $32,1 \%$, mientras que la participación de los alumnos pertenecientes al primer quintil aumenta de $4,5 \%$ a $10,4 \%$.

Gráfico 8. Distribución de alumnos causantes de AFI según quintil de ingreso per cápita del hogar*

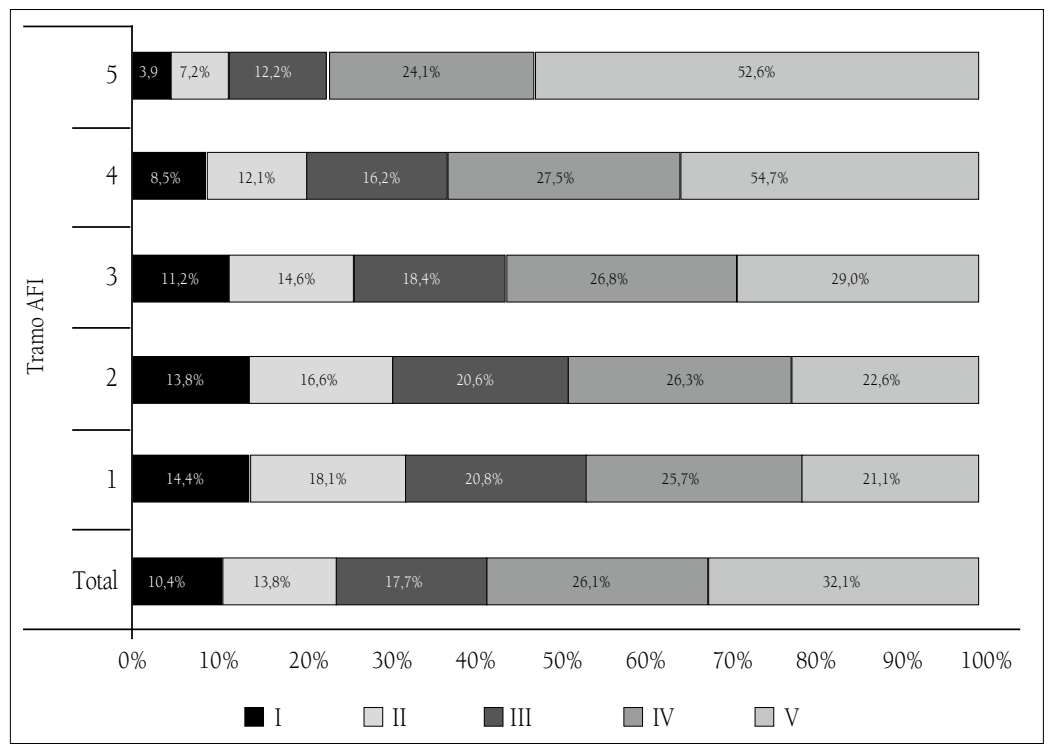

Fuente: elaboración propia sobre la base de PSU 2009.

* Corresponde al ingreso de los hogares declarados en el Formulario Único de Acreditación Socioeconómica (FUAS). 